\title{
Targeted Observations of Tropical Cyclone Movement Based on the Adjoint-Derived Sensitivity Steering Vector
}

\author{
Chun-Chieh Wu, Jan-Huey Chen, Po-Hsiung Lin, and Kun-Hsuan Chou \\ Department of Atmospheric Sciences, National Taiwan University, Taipei, Taiwan
}

(Manuscript received 16 May 2005, in final form 30 October 2006)

\begin{abstract}
Since 2003, a field program has been conducted under the name of Dropwindsonde Observations for Typhoon Surveillance near the Taiwan Region (DOTSTAR). As the name DOTSTAR suggests, targeted observation is one of its key objectives. The prerequisite for designing the observing strategy is to identify the sensitive areas, which would exert great influence on the results of numerical forecast or the extent of the forecast error.

In addition to various sensitivity products already adopted in DOTSTAR, a new way to identify the sensitive area for the targeted observation of tropical cyclones based on the fifth-generation Pennsylvania State University-National Center for Atmospheric Research (NCAR) Mesoscale Model (MM5) is proposed in this paper. By appropriately defining the response functions to represent the steering flow at the verifying time, a simple vector, adjoint-derived sensitivity steering vector (ADSSV), has been designed to demonstrate the sensitivity locations and the critical direction of typhoon steering flow at the observing time. Typhoons Meari and Mindulle of 2004 have been selected to show the use of ADSSV. In general, unique sensitive areas $36 \mathrm{~h}$ after the observing time are obtained.

The proposed ADSSV method is also used to demonstrate the signal of the binary interaction between Typhoons Fungwong and Fengshen (2002). The ADSSV is implemented and examined in the field project, DOTSTAR, in 2005 as well as in the surveillance mission for Atlantic hurricanes conducted by the Hurricane Research Division. Further analysis of the results will be vital to validate the use of ADSSV.
\end{abstract}

\section{Introduction}

A typhoon is one of the most destructive disasters and therefore causes great loss of life and property year by year in Taiwan. Since 2003, in order to promote the typhoon research, the National Science Council (NSC) in Taiwan has granted funding to the project, Dropwindsonde Observations for Typhoon Surveillance near the Taiwan Region (DOTSTAR; Wu et al. 2005), which is an interdepartmental and international project between Taiwan and the United States. The principal part of the project is the use of the airborne global positioning system (GPS) dropwindsondes released from the jet aircraft flying above 42000 feet in the environment of a tropical cyclone approaching near Taiwan. For DOTSTAR, targeted observations constitute one of the most critical objectives. To use the limited

Corresponding author address: Chun-Chieh Wu, Department of Atmospheric Sciences, National Taiwan University, No. 1, Sec. 4, Roosevelt Rd., Taipei 106, Taiwan.

E-mail: cwu@typhoon.as.ntu.edu.tw aircraft resources to maximize the possible improvement of numerical forecast, targeted observation strategy, which includes the flight route and the dropwindsondes deployment locations, must be developed before the flight mission. The basis for formulating the observation strategy is to identify the sensitive areas, which would considerably influence the results and sometimes even accuracy of the numerical forecast.

Until 2004, three sensitivity products have been used to determine the observation strategy for DOTSTAR. These products are derived from three distinct techniques. The first technique is the deep-layer mean (DLM) wind variance. It is one of the deep-layer steering flows based on the National Centers for Environmental Predictions (NCEP) Global Ensemble Forecasting System (EFS; Aberson 2003), where areas with the largest forecast DLM wind bred vectors represent the sensitive region at the observing time. The second technique is the ensemble transform Kalman filter (ETKF; Majumdar et al. 2002). This technique is able to predict the reduction in forecast error variance for feasible deployment of targeted observations based

DOI: $10.1175 / J A S 3974.1$ 
on the 40-member NCEP EFS. The third is the singular vector (SV) technique (e.g., Palmer et al. 1998; Peng and Reynolds 2006). It maximizes the growth of a total energy or kinetic energy norm using the forward and adjoint models of the Navy Operational Global Atmospheric Prediction System (NOGAPS; Rosmond 1997; Gelaro et al. 2002).

As mentioned above, the ETKF and SV products are derived from the (total) energy or kinetic energy norm. For the DLM wind variance, high sensitivity has a tendency to appear around the storm region as there is generally higher ensemble variability associated with small displacement of strong cyclonic winds near the core. Therefore, none of the above techniques for targeted observations is directly related to the motion (steering flow) of the tropical cyclone.

Theoretical work to determine a targeted observation strategy for better predicting the tropical cyclone track is inadequate in the literature (save the notable study of Rohaly et al. 1998). Along with the progress in DOTSTAR, we propose a new method to determine the sensitive area for targeted observation based on the adjoint sensitivity (Zou et al. 1997; Kleist and Morgan 2005a) to verify the sensitive areas with respect to the typhoon steering flow. A response function is designed to represent the steering flow at the verifying time, and to assess the adjoint sensitivity with respect to such response functions. A simple parameter is also proposed to interpret the sensitivity with clear physical meanings. The methodology and experiment design of this study are presented in section 2 . Section 3 provides the descriptions and adjoint-derived sensitivity steering vector (ADSSV) results of Typhoons Mindulle and Meari. In section 4, the above sensitivity parameter is used to validate the binary interaction between Typhoons Fungwong and Fengshen. Examination of the linear assumption in the adjoint modeling system is presented in section 5 . The summary and future prospects are shown in section 6 .

\section{Methodology and experiment design}

Scientists working on targeted observations are always interested in knowing the sensitivity of the specific model output to the initial input variables and thus in identifying where to make adapted observations to improve weather forecast of specific interests. To identify the sensitivity, a basic and direct practice would be to change the input variables and to examine the response of the model output. However, this is rather inefficient since there is an infinite amount of input variables to be perturbed. Errico (1997) has shown that the adjoint model can be a powerful tool for many studies that

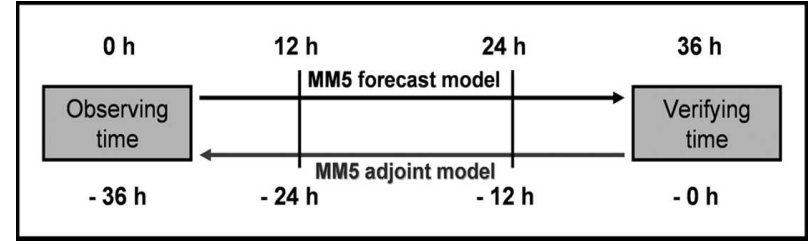

FIG. 1. The design of the forward and backward model integrations. The negative sign before the time indicates the backward integration (using the negative time step) associated with the adjoint model.

require an estimate of sensitivity of model output with respect to input. In other words, by defining the response function, as a function of model output variables, of research interests, one can use the adjoint model to effectively calculate the sensitivity of response function to the model input variables $\left(\partial R / \partial x_{\text {in }}\right)$ (Kleist and Morgan 2005a).

Our study utilizes a component of the fifthgeneration Pennsylvania State University-National Center for Atmospheric Research (NCAR) Mesoscale Model (MM5; Zou et al. 1997; Wu et al. 2006), which was used by Kleist and Morgan (2005a) to investigate a snowstorm with a poor forecast. This system includes the nonlinear MM5, its tangent linear model (TLM), and corresponding dry-physics adjoint model. The domain for the nonlinear and adjoint models we use is a $60-\mathrm{km}, 85 \times 115$ (latitude by longitude) horizontal grid, with 20 sigma levels in the vertical. The nonlinear integrations use the following physical parameterizations: the Grell cumulus parameterization, the bulk PBL scheme, the simple cooling radiation scheme, and the stable precipitation where the large-scale saturation is removed and rained out immediately but without rain evaporation or explicit cloud prediction. The TLM and adjoint integrations use the same parameterizations (or their adjoints), but the effect of moisture is neglected. The initial and boundary conditions are from the NCEP Global Forecasting System (GFS) global analysis $\left(1^{\circ} \times\right.$ $\left.1^{\circ}\right)$ interpolated to the MM5 grids.

The forward and backward integrations were executed by the MM5 forecast model and the adjoint model, respectively, as indicated in Fig. 1. The negative sign before the time indicates the backward integration (using the negative time step) associated with the adjoint model.

The work is aimed to identify the sensitive areas at the observing time, which will affect the steering flow of the typhoon at the verifying time. It has generally been proposed that tropical cyclone motion is governed by the environmental steering flow (Chan and Gray 1982), generally defined as the tropospheric average wind within the inner $5^{\circ}$ or $7^{\circ}$ latitude radius. Neumann 
(1979) showed that the steering concept can account for about $80 \%$ of the variability in the 24 -h tropical cyclone motion. Therefore, we define the response function(s) as the deep-layer mean wind within the verifying area. A square of $600 \mathrm{~km} \times 600 \mathrm{~km}$, centered around the MM5-simulated storm location (Fig. 3) at the verifying time, is used to calculate the background steering flow. Two responses functions are then defined: $R_{1}$, the 850 300-hPa deep-layer area average (Wu et al. 2003) of zonal component $(u)$, and $R_{2}$, the average of meridional component $(v)$ of the wind vector; that is,

$$
\begin{aligned}
& R_{1} \equiv \frac{\int_{850 \mathrm{hPa}}^{300 \mathrm{hPa}} \int_{A} u d x d y d p}{\int_{850 \mathrm{hPa}}^{300 \mathrm{hPa}} \int_{A} d x d y d p} \text { and } \\
& R_{2} \equiv \frac{\int_{850 \mathrm{hPa}}^{300 \mathrm{hPa}} \int_{A} v d x d y d p}{\int_{850 \mathrm{hPa}}^{300 \mathrm{hPa}} \int_{A} d x d y d p} .
\end{aligned}
$$

In other words, by averaging out the axisymmetric component of the strong cyclonic flow around the storm center, the vector of $\left(R_{1}, R_{2}\right)$ represents the background steering flow across the storm center at the verifying time. It should be noted that a wind vector $\left(R_{1}, R_{2}\right)$ is totally different from the kinetic energy norm stated above.

First, $d R_{1} / d u$ and $d R_{1} / d v\left(d R_{2} / d u\right.$ and $\left.d R_{2} / d v\right)$ are calculated to indicate the sensitivity of the zonal (meridional) component of the steering flows at the verifying time to the flow field at the observing time. To show a general sensitivity to the wind field, we combine the sensitivity of $R_{1}\left(R_{2}\right)$ to $u$ and the sensitivity of $R_{1}\left(R_{2}\right)$ to $v$ to obtain the sensitivity of $R_{1}\left(R_{2}\right)$ to the vorticity field $\left(\partial R_{1} / \partial \varsigma\right.$ and $\left.\partial R_{2} / \partial \varsigma\right)$ and (Kleist and Morgan 2005a). In order to interpret the sensitivity with clear physical meanings, we design a unique new parameter, ADSSV, to identify the sensitive areas at the observing time to the steering flow at the verifying time. The ADSSV with respect to the vorticity field (s) can be shown as

$$
\operatorname{ADSSV} \equiv\left(\frac{\partial R_{1}}{\partial \varsigma}, \frac{\partial R_{2}}{\partial \varsigma}\right)
$$

where, at a given point, the magnitude of ADSSV, which is contributed by the sensitivities of the two response functions (referred to as the typhoon steering flow) to the vorticity field, indicates the extent of the sensitivity. Meanwhile, the direction of the ADSSV,

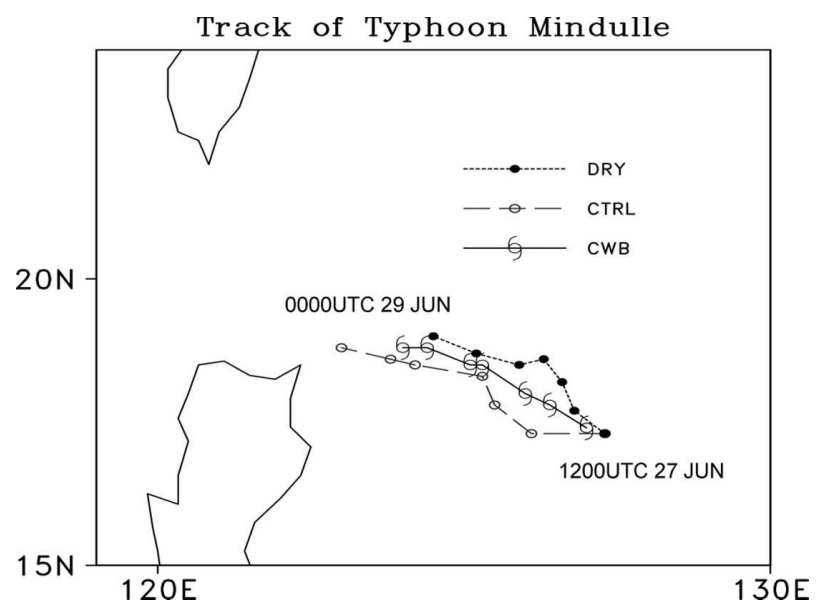

FIG. 2. Track of Mindulle from 1200 UTC 27 Jun to 0000 UTC 29 Jun from the MM5 forecasts (CTRL and DRY) and the besttrack analysis of CWB.

which is controlled by the relative magnitude of two sensitivities, represents the direction toward which the steering flow would increase with respect to a vorticity perturbation placed at that point. For example, if at a given forecast time at one particular grid point the ADSSV vector points to the east, an increase in the vorticity at the very point at the observing time would be associated with an increase in the eastward steering flow of the storm at the verifying time.

\section{Results}

Typhoon Mindulle in 2004, one of the cases observed in DOTSTAR, is chosen as a test case to examine the proposed new method for targeted observations based on the adjoint sensitivity. Note that Mindulle is the sole case out of the ten DOTSTAR cases in 2004 where dropwindsonde data assimilated into the NCEP GFS model did not help improve the track forecasts (Wu et al. 2007). The study is based on a 36-h MM5 simulation initialized at 1200 UTC 27 June 2004 (denoted as CTRL). Figure 2 shows that the model storm in CTRL moves along (but slightly faster than) the best track from the Central Weather Bureau (CWB) of Taiwan.

Based on Eq. (1), we first show that the background steering flow $\left(R_{1}, R_{2}\right)$ at the verifying time in MM5 is $\left(-1.25,-0.24 \mathrm{~m} \mathrm{~s}^{-1}\right)$, which is consistent with the model's slow westward movement of Mindulle at the verifying time (i.e., 0000 UTC 29 June 2004; see Fig. 2 for the modeled storm track in CTRL). In this paper, only the sensitivity products at $700 \mathrm{hPa}$ are shown. In general, the results of the sensitivity patterns at 850 or 500 $\mathrm{hPa}$ are qualitatively consistent with each other.

As expected, the sensitivity (i.e., gradient) of $R_{1}$ to $u$ 

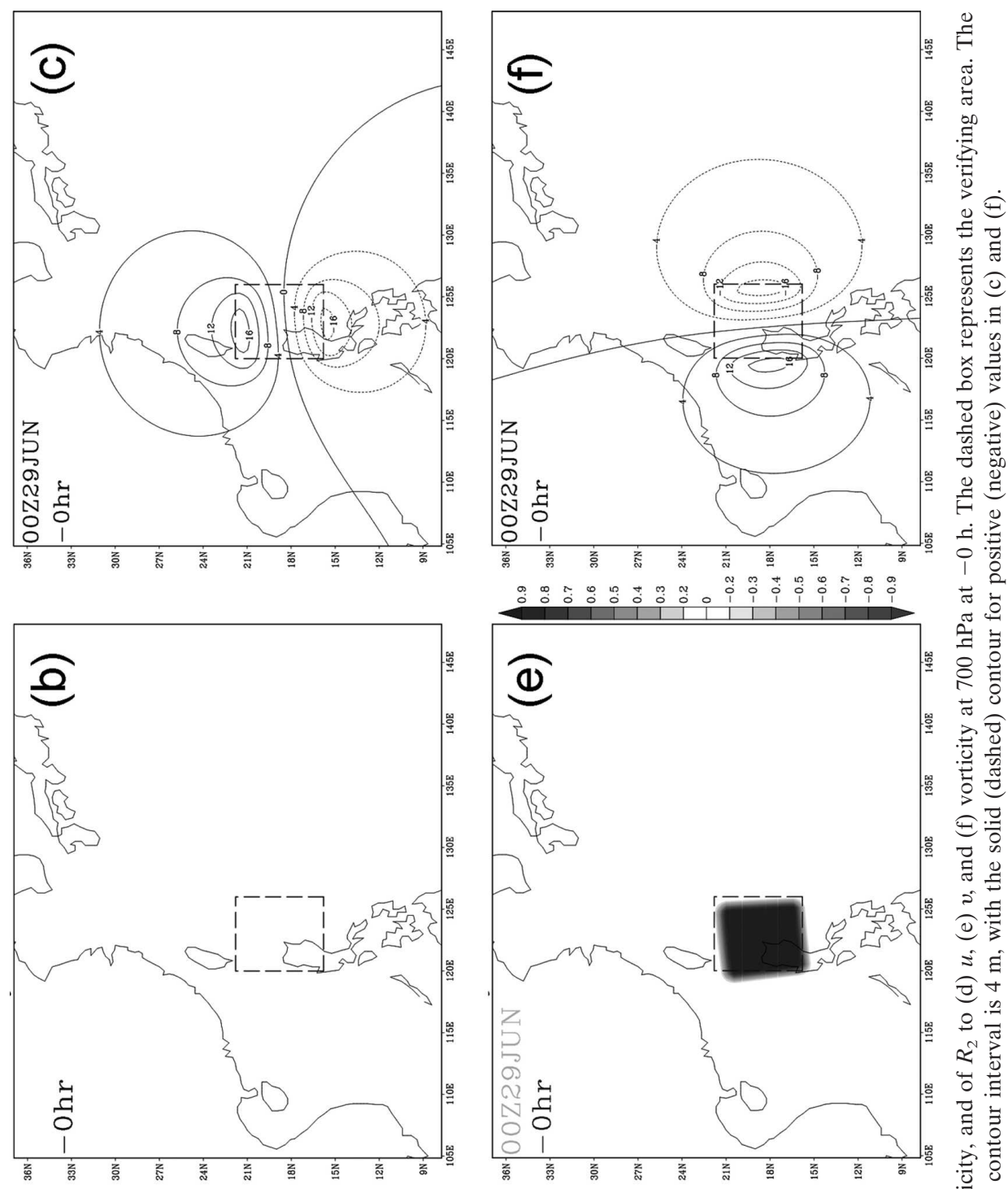

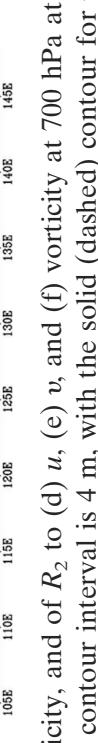

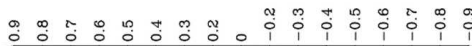
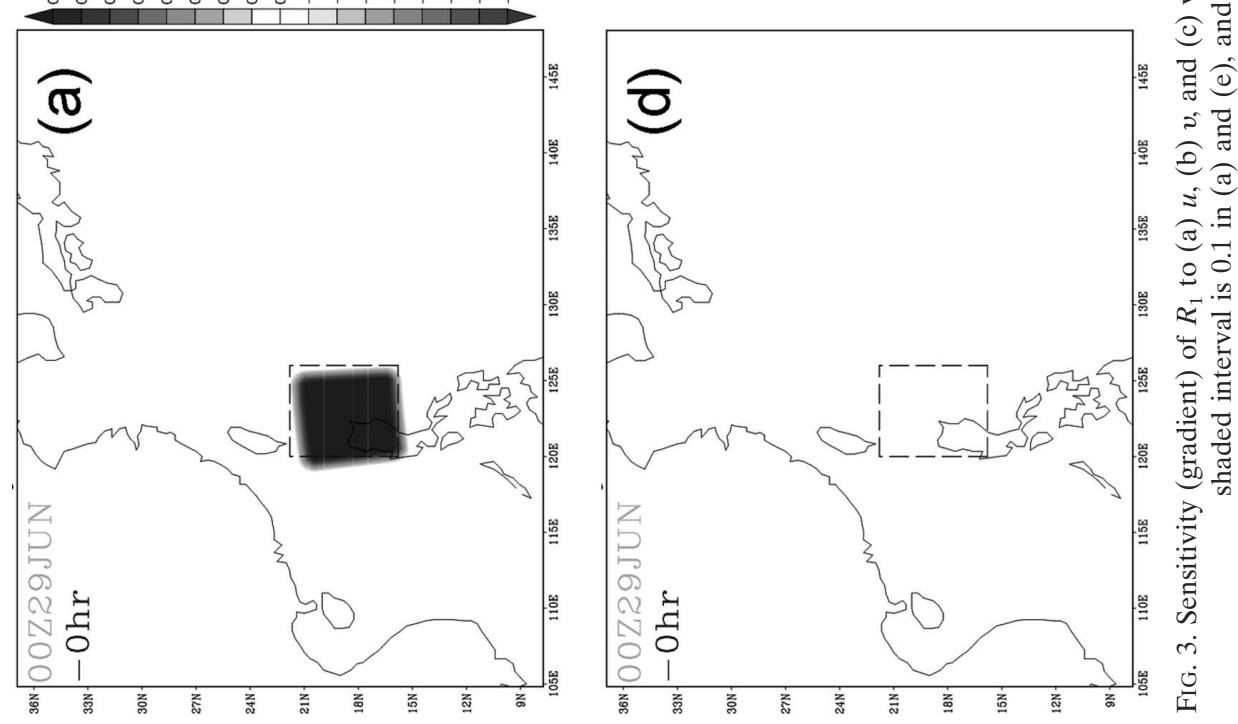

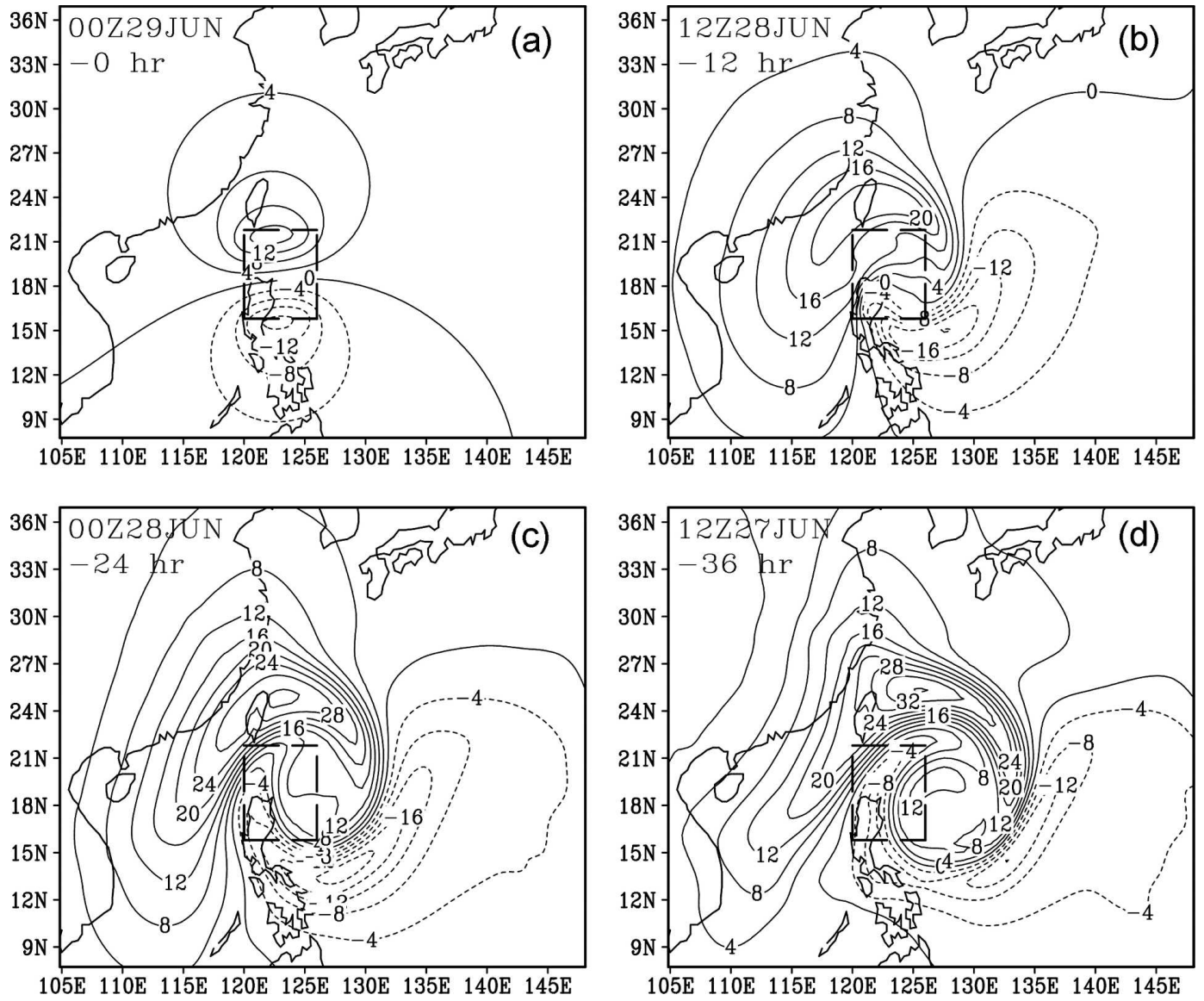

FIG. 4. The evolution of sensitivity of $R_{1}$ with respect to the vorticity field at $700 \mathrm{hPa}$ (contour interval of $4 \mathrm{~m}$ ).

The dashed box represents the verifying area.

$\left(\partial R_{1} / \partial u\right)$ at $-0 \mathrm{~h}$ (the initial time of adjoint model) shows a response uniformly distributed over the verifying area (Fig. 3a), while there is no sensitivity of $R_{1}$ to $v\left(\partial R_{1} / \partial v\right)$ (Fig. 3b). To show a general sensitivity to the wind field, we combine the sensitivity of $R_{1}$ to $u$ and the sensitivity of $R_{1}$ to $v$ to obtain the sensitivity of $R_{1}$ to the vorticity field $\left(\partial R_{1} / \partial \varsigma\right)$ (Kleist and Morgan 2005a). Again, as expected, a dipolar pattern at $-0 \mathrm{~h}$ (Fig. 3c) is found; that is, a positive (negative) vorticity perturbation to the north (south) of the verifying area is associated with a cyclonic (anticyclonic) circulation and thus leads to an increase in $R_{1}$ (the zonal component of the mean steering flow). Meanwhile, the sensitivity of $R_{2}$ to $u, v$, and the vorticity field (Figs. 3d,e,f) also reveals comparable information. In all, Figs. 3 c,f succinctly show the sensitivity of $R_{1}$ and $R_{2}$ to the flow field with clear physical meanings.

The evolutions of the sensitivity of $R_{1}$ and $R_{2}$ to the vorticity field are shown in Figs. 4 and 5. The sensitive areas spread from the margin of the verifying area to the outer region as the adjoint model is integrated backward in time. At $-36 \mathrm{~h}$ (the observing time, 1200 UTC 27 June), the large gradient (and thus the high sensitivity) areas are located in the east and north of the verifying area, and the sensitivity is found to be higher in $R_{2}$ than in $R_{1}$. This means that vorticity perturbations in those large gradient (sensitive) areas at 1200 UTC 27 June will affect the steering flow of Typhoon Mindulle at 0000 UTC 29 June, particularly the meridional component of the steering flow.

As shown in Eq. (2), we can combine the result of Figs. 4 and 5 to obtain the evolution of ADSSV with respect to the vorticity field (Fig. 6). Figure 6 clearly shows that the vectors rotate around the verifying area at $-0 \mathrm{~h}$. As the adjoint model integrates backward in time, these vectors evolve and expand outward, with longer vectors (i.e., higher sensitivity) mostly extending at about $800-1300 \mathrm{~km}$ from the north to the east of the center of verifying area.

In this experiment, we have only demonstrated the ADSSV at one single verifying time. Nevertheless, since the tropical cyclone's final position is affected by 

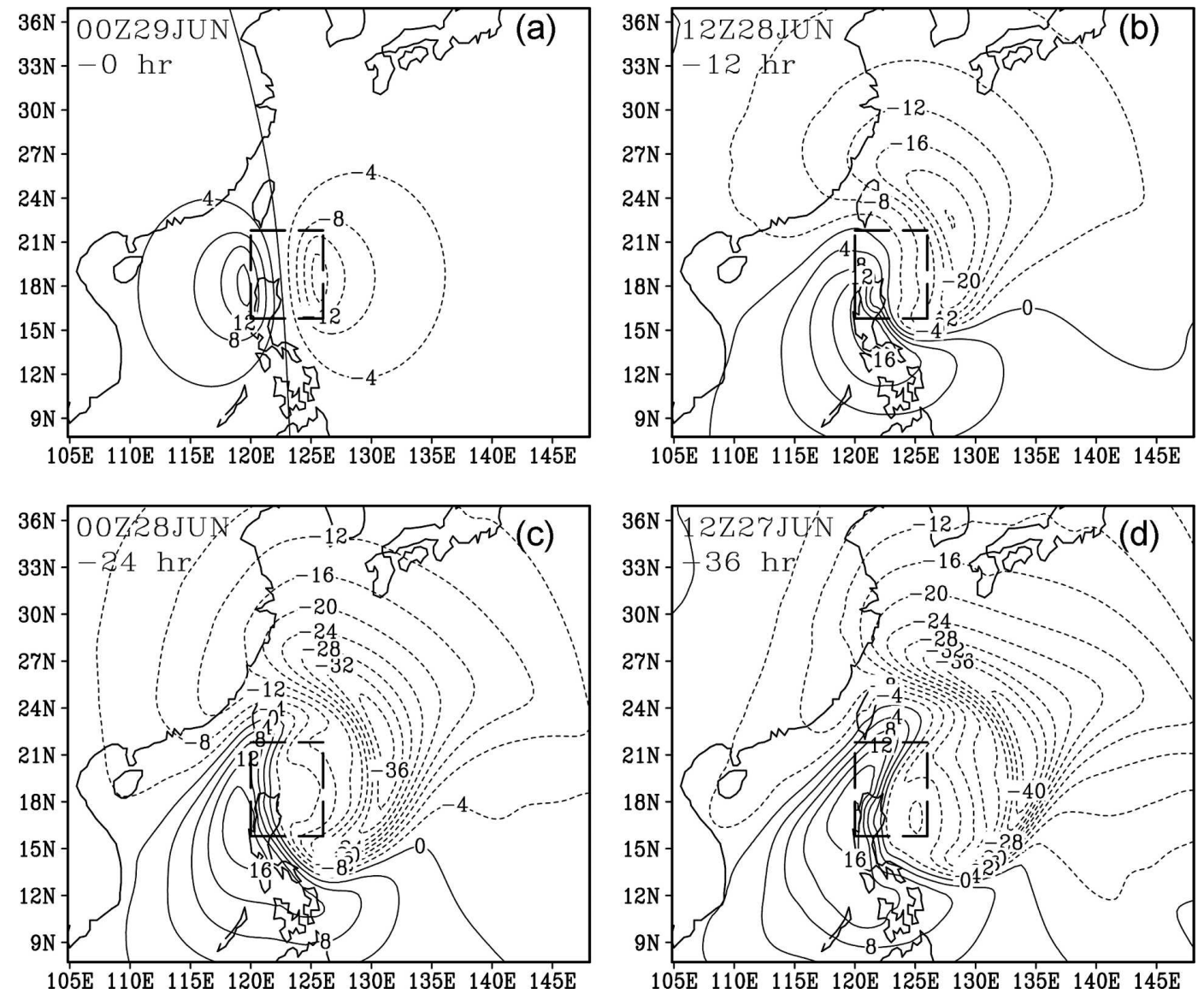

FIG. 5. The evolution of sensitivity of $R_{2}$ with respect to the vorticity field at $700 \mathrm{hPa}$ (contour interval of $4 \mathrm{~m}$ ). The dashed box represents the verifying area.

the steering flow before and up to the verifying time, it is critical to also calculate the adjoint sensitivity for different verifying times along the storm track; thus the impact of the targeted observations on the entire tropical cyclone track can be better assessed. Other than performing the 36-h forward model simulation and 36-h backward adjoint integration as shown above, for the same starting (observing) time, we have also conducted the 24- and 12-h forward and backward integrations to obtain the respective ADSSVs associated with the response function $\left(R_{1}, R_{2}\right)$ based on the model-predicted storm location at each verifying time. By combining all the ADSSV plots at 12,24 , and $36 \mathrm{~h}$ into the observing time in one figure (see Fig. 7), we can clearly identify the sensitive regions that affect the steering flows at 12 , 24 , and $36 \mathrm{~h}$, respectively, and thus the regions for targeted observations that improve the typhoon track up to the verifying time.

As such, the ADSSV with respect to the vorticity field at 12 (in green), 24 (in red), and $36 \mathrm{~h}$ (in blue) is plotted in Fig. 7, superposed with the geopotential height field at $700 \mathrm{hPa}$ at the observing time and the deployed locations of the dropwindsondes in DOTSTAR. Note that the ADSSV for different verifying times more or less corresponds to one another. Figure 7, in which the vectors in regions of large ADSSV mainly point southward, indicates the southward component of steering flow strengthens (weakens) with the increase (decrease) in the vorticity in those sensitive areas. Physically, these vectors are located at the edge of the subtropical high, where, if the subtropical high strengthens (i.e., with decreased vorticity), the northward steering increases. The results also show that the extent of the subtropical high is crucial to determining Mindulle's northward deflection as observed at the verifying time.

Besides the ADSSV with respect to the vorticity field, we also calculate the ADSSV with respect to the divergence field, which is

$$
\left(\frac{\partial R_{1}}{\partial D}, \frac{\partial R_{2}}{\partial D}\right)
$$



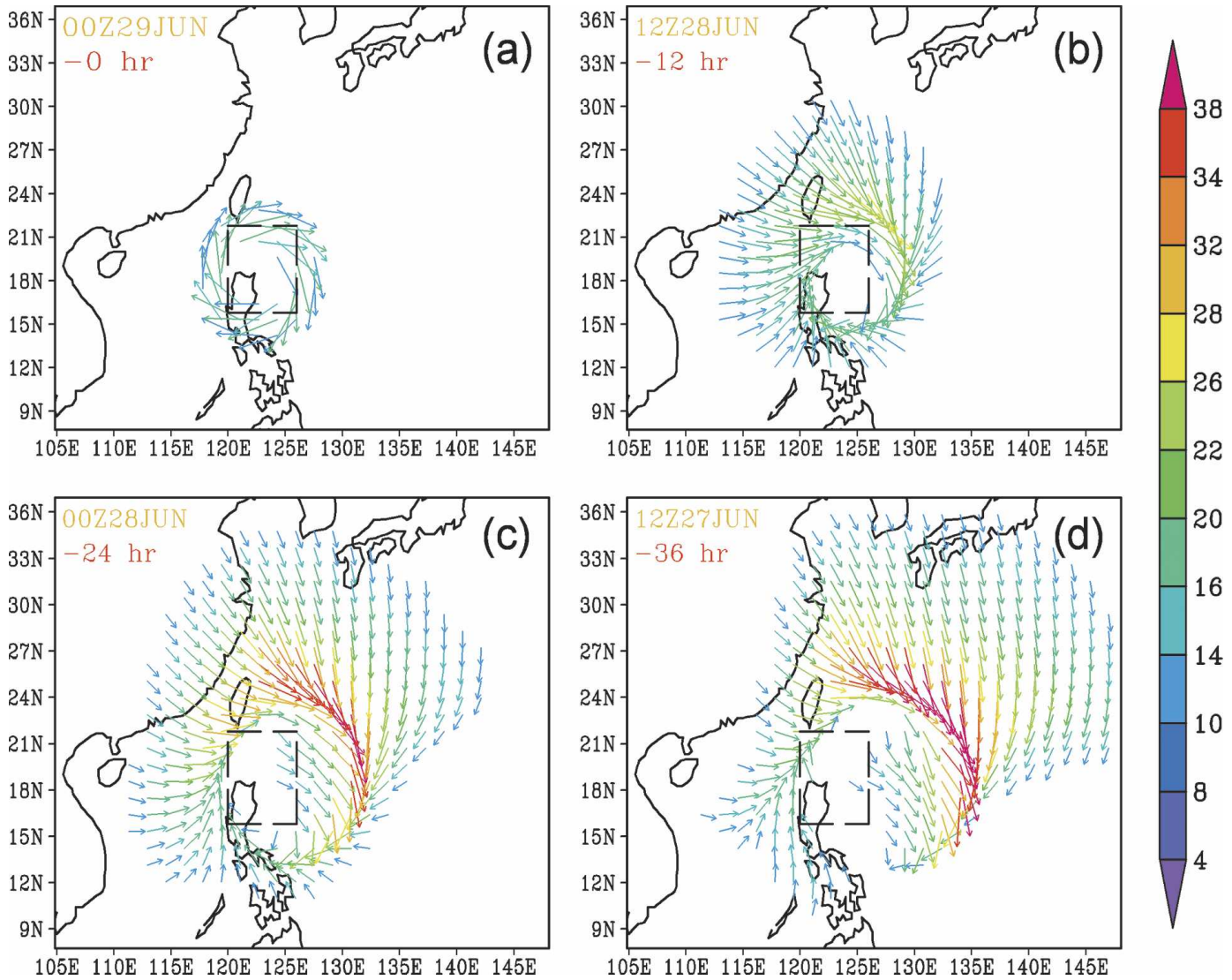

FIG. 6. The evolution of the ADSSV (magnitude of the vector given by the color bar scale to the right, unit: $\mathrm{m}$ ) with respect to the vorticity field at $700 \mathrm{hPa}$. The dashed box represents the verifying area.

where $D$ represents the divergence of the wind field. It is found that the sensitivity to the divergence field (Fig. 8 ) is one order magnitude smaller than that to the vorticity field. The above result indicates that the steering flow bears much larger sensitivity to the vorticity field than to the divergence field.

Note that the ADSSV shown above is a dimensional vector. To compare the relative importance of the ADSSV with respect to the vorticity and the divergence fields, the nondimensional ADSSV (ADSSV-ND) is also calculated. The ADSSV-ND with respect to vorticity and divergence fields can be defined as

$$
\left(\frac{\frac{\partial R_{1}}{\partial \varsigma}\left|\varsigma\left(t_{0}\right)\right|}{\left|R_{1}\right|}, \frac{\frac{\partial R_{2}}{\partial \varsigma}\left|\varsigma\left(t_{0}\right)\right|}{\left|R_{2}\right|}\right),
$$

and

$$
\left(\frac{\frac{\partial R_{1}}{\partial \varsigma}\left|D\left(t_{0}\right)\right|}{\left|R_{1}\right|}, \frac{\frac{\partial R_{2}}{\partial \varsigma}\left|D\left(t_{0}\right)\right|}{\left|R_{2}\right|}\right),
$$

respectively, where $t_{0}$ is the initial time of the forecast model. It can be demonstrated (figures not shown) that the ADSSV-ND with respect to the vorticity field is still much larger (by as much as two orders of magnitude) than the ADSSV-ND with respect to the divergence field. Hereafter, without loss of generosity, this study will mainly focus on the ADSSV with respect to the vorticity field, while neglecting the ADSSV with respect to the divergence field.

Meanwhile, it is found that DOTSTAR's dropsondes for the case of Mindulle are not deployed in the highsensitivity region in the ADSSV plot in Fig. 7. On the other hand, the sensitive regions in Fig. 7 are quite different from those indicated by three other independent sensitivity products (figures not shown) currently used for planning the real-time targeted observations for DOTSTAR (Wu et al. 2005). Subsequent research is needed to assess such differences and to evaluate the strength and weakness of each product (e.g., Majumdar et al. 2006; Etherton et al. 2006).

Besides Mindulle, Typhoon Meari (2004) is another observed case in DOTSTAR. It is shown that the drop- 


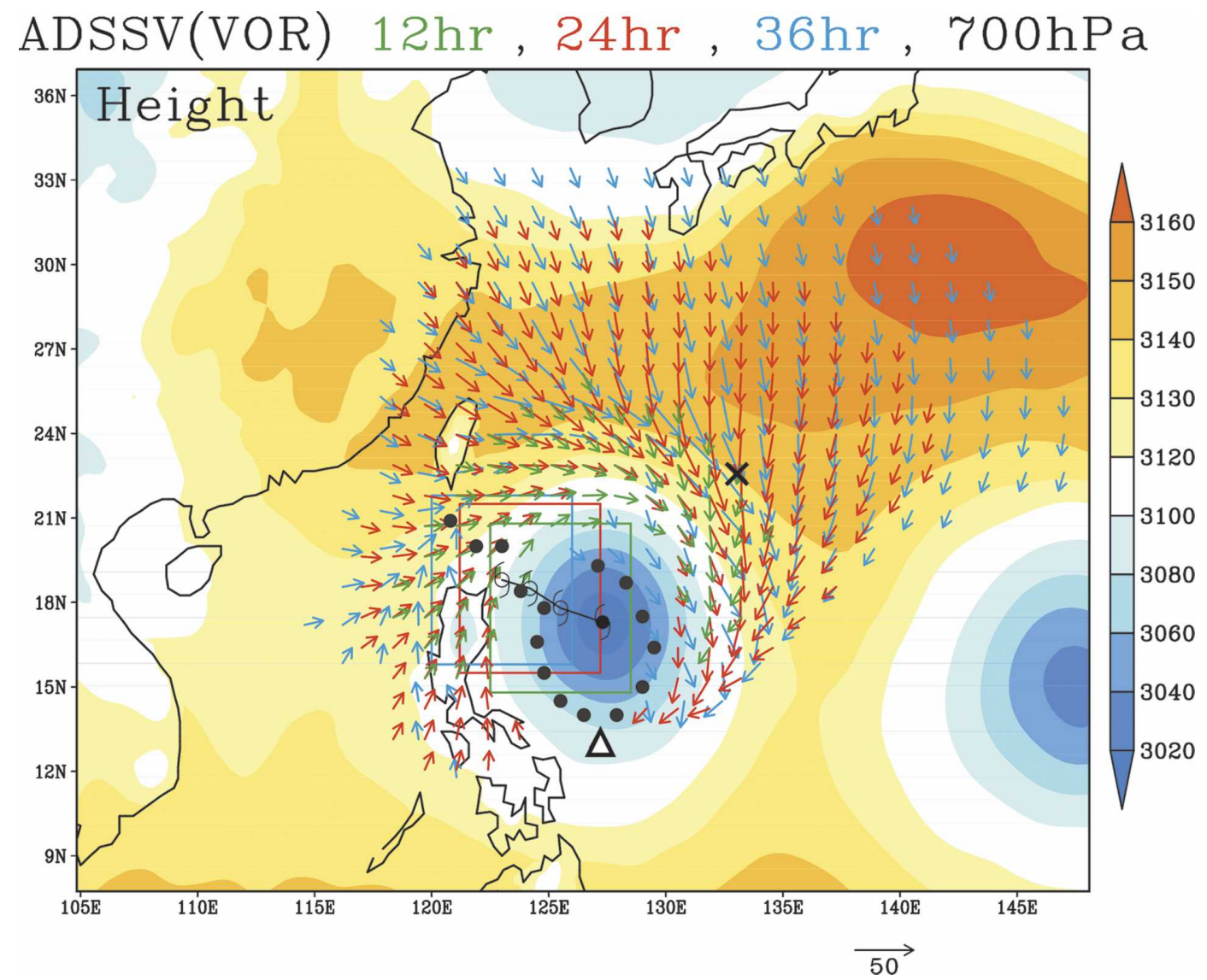

FIG. 7. ADSSV with respect to the vorticity field at $700 \mathrm{hPa}$ at 12 (green), 24 (red), and $36 \mathrm{~h}$ (blue) as the verifying time, superposed with the geopotential height field (magnitude scaled by the color bar to the right, unit: $\mathrm{m}$ ) at $700 \mathrm{hPa}$ and the deployed locations of the dropsondes in DOTSTAR (brown dots). The scale of the ADSSV vector is indicated as the arrow to the lower right (unit: $\mathrm{m}$ ). The 36-h model-predicted track of Mindulle is indicated with the typhoon symbols in red for every $12 \mathrm{~h}$. The three square boxes represent the verifying areas at three different verifying times. The $\times$ and the $\triangle$ show the grid locations where the wind perturbation is added at the initial time for the linearity tests shown in Figs. 13 and 14.

sonde data from DOTSTAR has a very positive impact on the 72-h track forecasts of Meari in the NCEP GFS model (Wu et al. 2007). Here we also study the ADSSV associated with Meari from a 36-h MM5 simulation initialized at 1200 UTC 25 September. Similar to the plots in Fig. 7, the ADSSV plots with respect to the vorticity field at 12 (in green), 24 (in red), and $36 \mathrm{~h}$ (in blue), respectively, are shown in Fig. 9. Consistent with the case of Mindulle in Fig. 7, the sensitivity areas as depicted in the ADSSV for Meari at different verifying times in Fig. 9 are collocated with one another. These vectors mostly extend at about $300-600 \mathrm{~km}$ from the north to the east of the storm center. It is obvious most of the ADSSVs point southward, indicating the higher sensitivity in affecting the meridional component of the steering flow in these areas. As in Typhoon Mindulle, these sensitive areas are located at the edge of the 700$\mathrm{hPa}$ subtropical high, implicating that the variation of the subtropical high is important to the track of Typhoon Meari.

Different from Mindulle, the deployed locations of DOTSTAR's dropwindsondes in Meari well match the high ADSSV region in Fig. 9. Work is ongoing to help investigate the impact of the dropwindsonde data on the forecast models and identify their relation with the ADSSV (e.g., Huang et al. 2006).

\section{A validation study of binary interaction}

\section{a. Synopsis}

In this section, Typhoons Fengshen and Fungwong in 2002, which exhibit a clear binary interaction (Yang and $\mathrm{Wu} 2004$ ), are chosen as a show case to validate whether the ADSSV can delineate the sensitivity of such an interaction process. Yang and $\mathrm{Wu}$ (2004) investigated this binary interaction from the potential vor- 

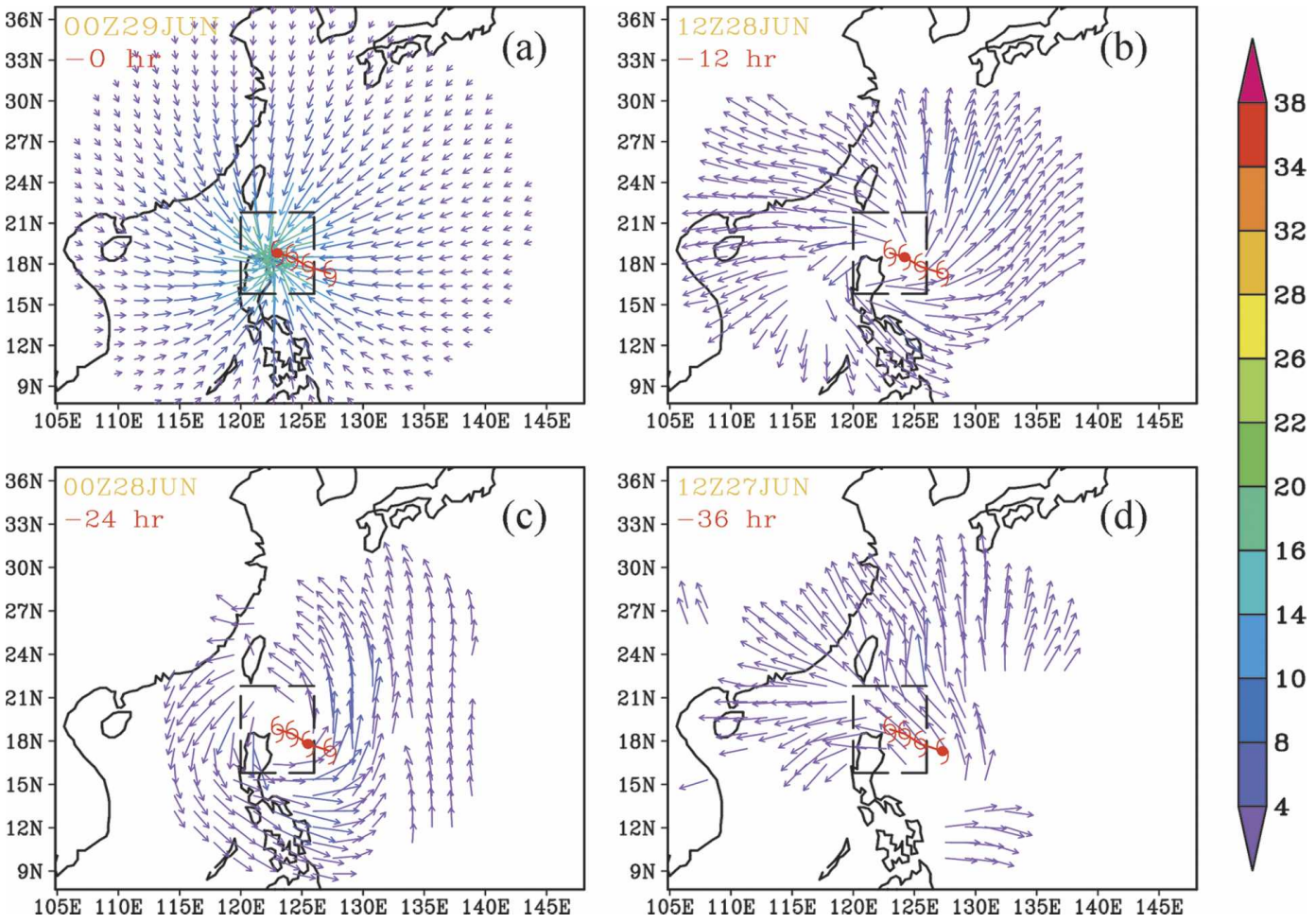

FIG. 8. Same as Fig. 6, but for the ADSSV respect to the divergence field. The dashed box represents the verifying area.

ticity diagnosis, and found obvious influence of Fengshen on Fungwong (one-way interaction) during 0000 UTC 23 July to 0000 UTC 25 July (figure not shown). In other words, the cyclonic looping of Fungwong (Fig. 10 ) is primarily induced by the flow associated with the stronger storm, Fengshen. On the other hand, the motion of Fengshen is only slightly affected by the flow associated with the weaker storm, Fungwong, while being mainly advected by the east-southeasterly steering flow associated with the subtropical high.

\section{b. ADSSV for binary interaction}

\section{1) ADSSV FOR THE TRACK PREDICTION OF FUNGWONG}

In this part, the ADSSV is calculated by the MM5 adjoint modeling system to demonstrate the sensitivity location that would affect Fungwong's steering flow. The MM5 forecast model is initialized at 0000 UTC 23 July, with the same domain settings as mentioned above. The forecast location of Fungwong in $48 \mathrm{~h}$ is set as the verifying area. The best-track analysis of CWB and the forecast tracks from MM5 are shown in Fig. 10.

Figure 11 shows the sensitive areas at the different forecast times, which will affect the steering flow of Fungwong at the verifying time. The locations of two typhoons can be recognized by the colors that represent the geopotential height field at $700 \mathrm{hPa}$ in these figures. Following the backward integration, the sensitive areas are always located around Fengshen, and the maximum ADSSV occurs between the two typhoons. It indicates that Fengshen is sensitive to the steering flow of Fungwong at the verifying time.

\section{2) ADSSV FOR THE TRACK PREDICTION OF FENGSHEN}

On the other hand, we can set the forecast location of Fengshen in $48 \mathrm{~h}$ as the verifying area. It is demonstrated in the ADSSV patterns in Fig. 12 that there is not much sensitivity near Fungwong that would affect the steering flow of Fengshen. Comparison of the ADSSV distribution in Fig. 11d and Fig. 12d apparently shows that Fengshen is sensitive to the steering flow associated with the circulation of Fungwong, but the sensitivity for Fungwong to the steering flow associated with Fengshen is rather insignificant. The maximum ADSSV of Fengshen is mainly located in the north of the storm itself. The above results are consistent with the PV analysis of Yang and $\mathrm{Wu}$ (2004), showing the nature of the one-way interaction between the two typhoons. Namely, the circulation associated with the 


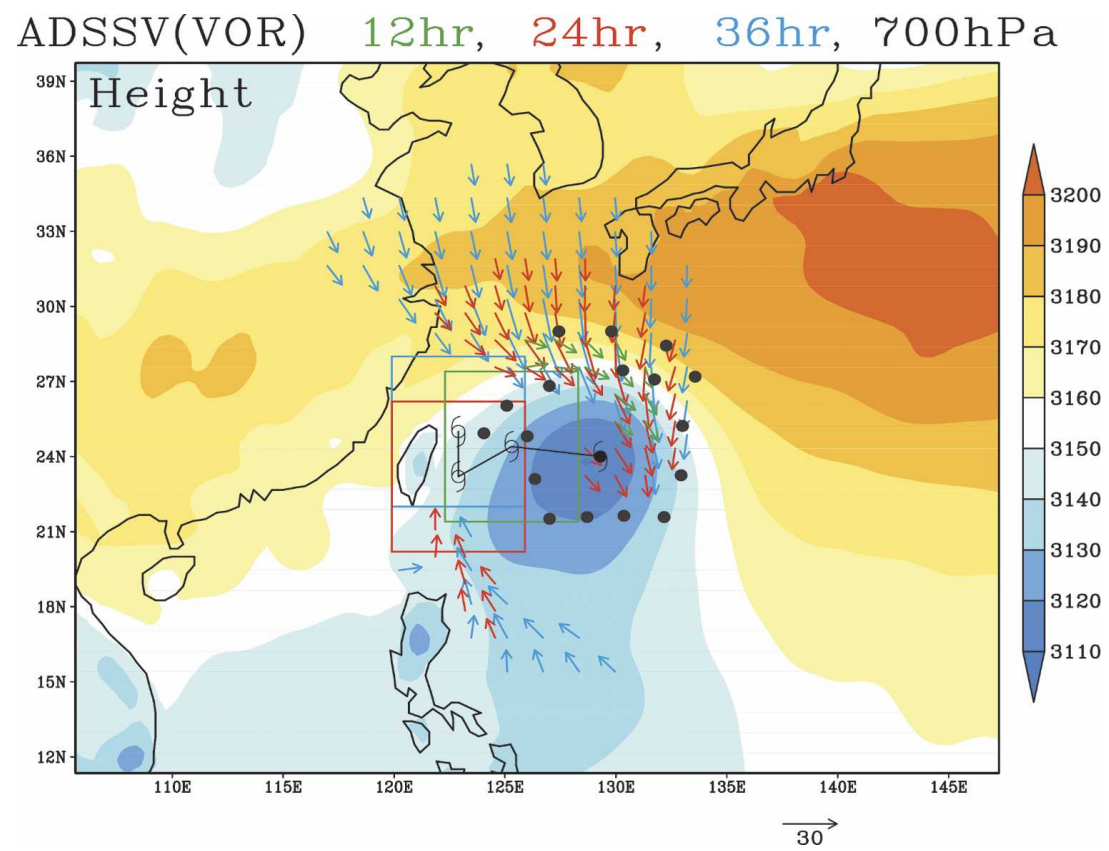

FIG. 9. Same as Fig. 7, but for Typhoon Meari. The scale of the ADSSV vector is indicated as the arrow to the lower right (unit: $\mathrm{m}$ ). The three square boxes represent the verifying areas at three different verifying times.

stronger Fengshen has greater influence on the track of the weaker Fungwong.

\section{Linearity test of the adjoint modeling system}

The adjoint model is designed based on the TLM, which is a linear assumptive model. As demonstrated in Kleist and Morgan (2005a), in order to validate this assumption, perturbations that evolve linearly via the TLM need to be compared with different fields obtained from two nonlinear model forecasts.

Three different groups of experiments are used to test the linear assumption for the case of Typhoon Mindulle. For the first, $1 \mathrm{~m} \mathrm{~s}^{-1}$ wind perturbation is added at a single grid point at the $\sigma=0.775$ level located within the most sensitive area of ADSSV for Typhoon Mindulle at $36 \mathrm{~h}$ (see the cross sign, $X$ in Fig. 7). Since both the TLM and the adjoint model we used contain no moist physics, as a fair test of the linear assumption, in the first experiment, the assigned perturbation is integrated in the TLM based on a basic state provided from the simulation of the nonlinear model with dry physics (denoted as TLM-D). Then two nonlinear simulations with dry physics are performed: one from the control simulation that creates the dry basic state (denoted as DRY) while the other simulation starts with the single-grid perturbation added to the above initial basic state. The difference of the two simulations (the former simulation subtracted by the latter) is denoted as NLM-D

As shown in Figs. 13 and 14, the time evolution of the wind perturbation (in both $u$ and $v$ ) of the TLM-D is nearly identical to that of NLM-D. It is clearly demonstrated that the linear assumption holds perfectly up to $36 \mathrm{~h}$ in the dry physics model (the result also remains robust at $48 \mathrm{~h}$; figures not shown). Indeed, when we choose the dry forward-model run (i.e., the DRY experiment) as the basic state, the obtained ADSSV pat-

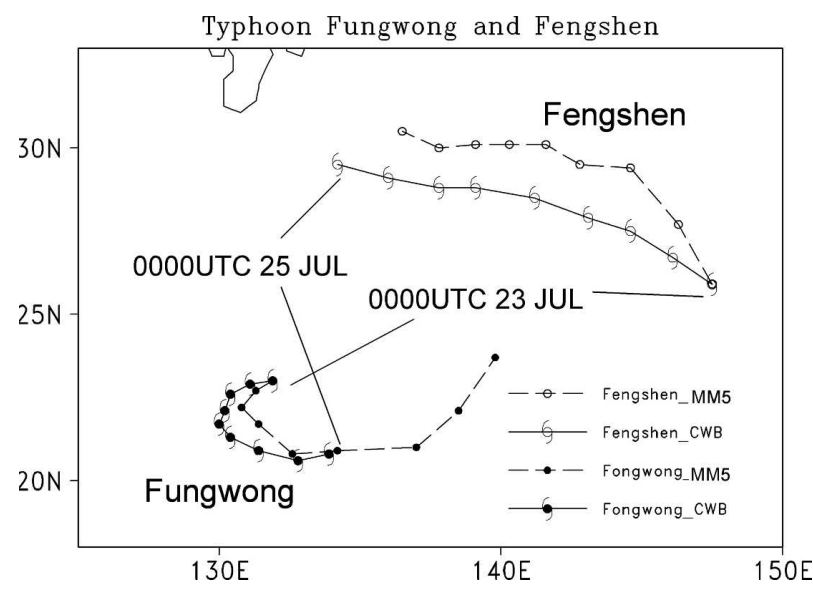

FIG. 10. Tracks of Fengshen and Fungwong from 0000 UTC 23 Jul to 0000 UTC 25 Jul from the MM5 forecast and the best-track analysis of CWB. 

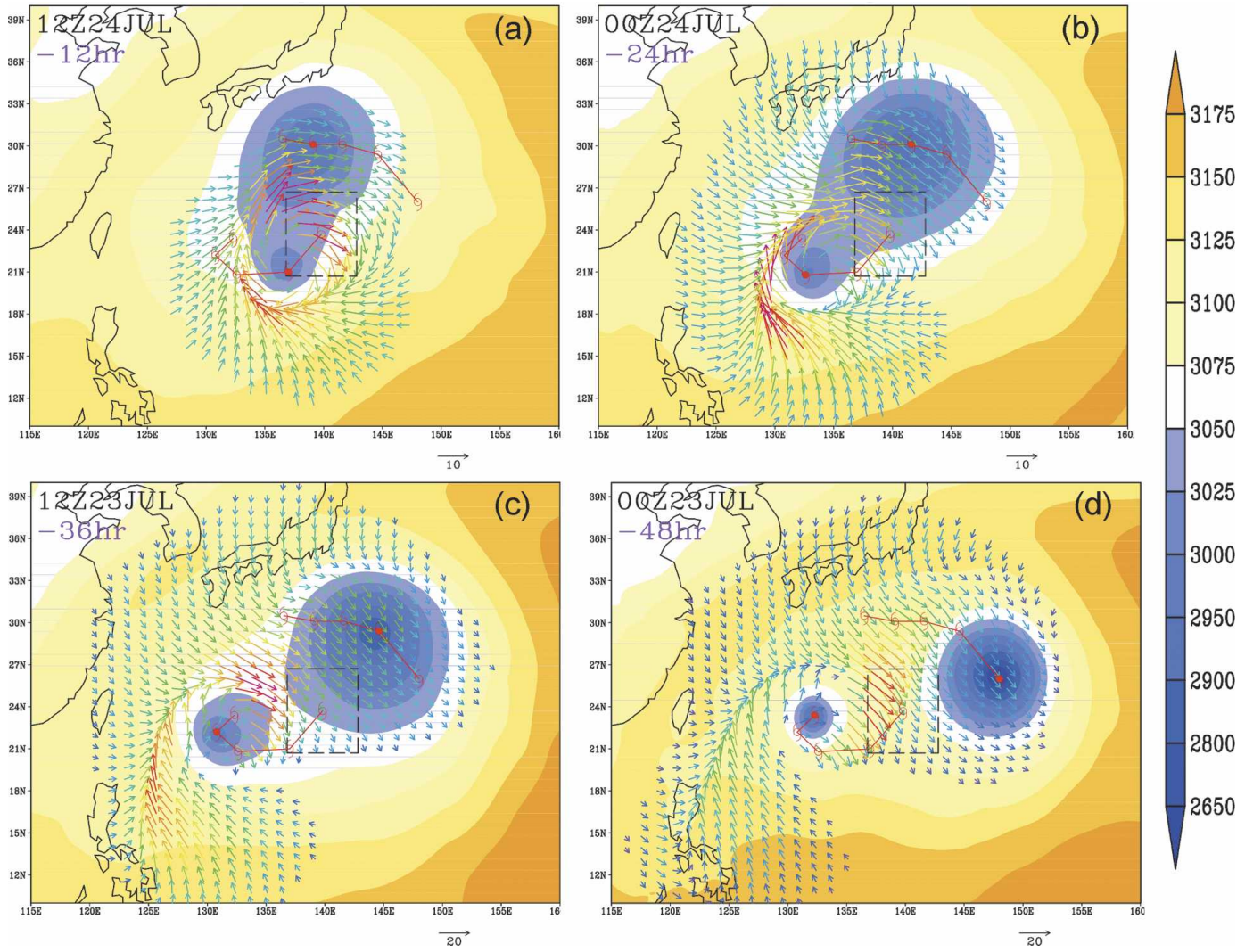

FIG. 11. The ADSSV at $700 \mathrm{hPa}$ at (a) -12 (36), (b) -24 (24), (c) $-36(12)$, and (d) -48 (0) $\mathrm{h}$ for $48 \mathrm{~h}$ as the verifying time, superposed with the geopotential height field (magnitude given by the color bar scale to the right, unit: gpm) at $700 \mathrm{hPa}$. The 48-h model-predicted tracks of Typhoon Fungwong and Typhoon Fengshen are indicated with the typhoon symbols in red for every $12 \mathrm{~h}$. The box with dashed line represents the verifying area with respect to Typhoon Fungwong. The scale of the ADSSV vector is indicated as the arrow to the lower right (unit: $\mathrm{m}$ ).

terns are nearly identical to the previous ones based on the moist basic state (figures not shown).

The second group is the same as the first, except that the perturbation is added at the nonsensitive grid location (as indicated by the triangle sign, $\triangle$ in Fig. 7). As shown in Fig. 15, the zonal component $(u)$ of the wind perturbation of the TLM evolves consistently with that of NLM-D, and the same for the meridional component ( $v$; figures not shown). Similar experiments have also been shown in other typhoon cases (such as Meari; figures not shown). The results all show that the linear assumption works well in all these dry experiments.

It is also worth noting that in the first group of experiments, the perturbation starts at the ADSSVsensitive location, and then it does grow and propagate into the verification region (i.e., the square box in Figs. 13 and 14) at $36 \mathrm{~h}$. On the other hand, for the second group of experiments, as shown in Fig. 15, the perturbation starts at a nonsensitive region, though it continues to grow and propagates eastward, but never shows any impact on the flow field in the verification region. This result appears consistent with our ADSSV theory, which reveals the sensitivity to the response function at the verification at the verification time.

One would naturally questions whether the dry assumption is valid in the typhoon simulation. As discussed in Peng and Reynolds (2006), we believe that the tropical cyclone movement is mainly controlled by the large-scale flow field, which is less directly affected or dominated by the moist physics. Indeed, Fig. 2 shows that the model storm from both the CTRL and DRY experiments experiences a consistently northwestward movement of Mindulle in their lifetime, though the DRY storm tends to move slower and show more northward and eastward track.

To evaluate the impact of the moist physics, the third group of experiments are performed based on the moist basic state in the TLM (denoted as TLM-M) and two nonlinear forecasts with moist physics (namely, one is CTRL, while the other is the same as CTRL, but with the single-grid perturbation added). The results (figure 

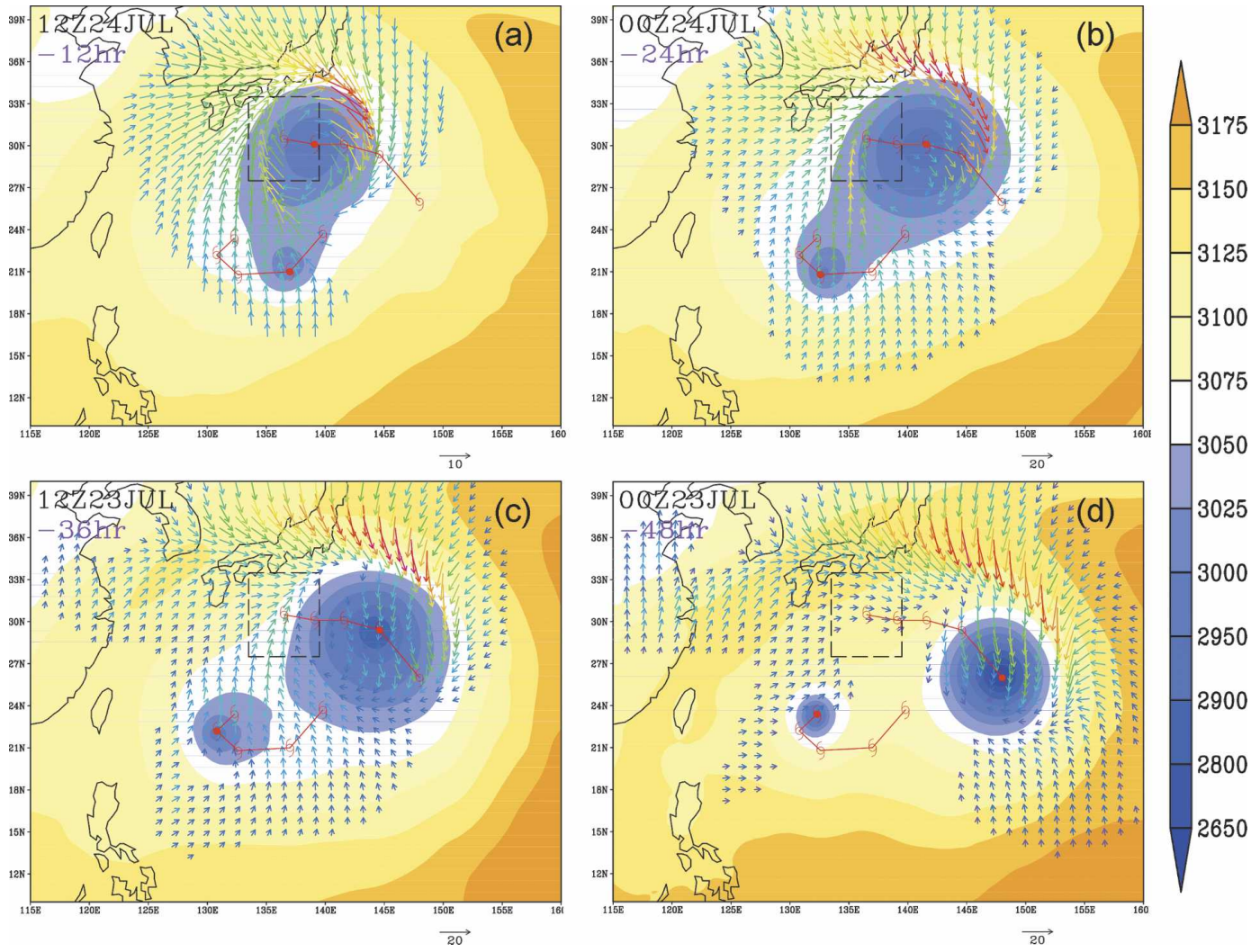

FIG. 12. Same as Fig. 9, but the box with the dashed line represents the verifying area with respect to Fengsheng. The scale of the ADSSV vector is indicated as the arrow to the lower right (unit: $\mathrm{m}$ ).

not shown) show that the 36-h perturbation of the TLM-M remains very similar to that of TLM-D. However, the difference of two nonlinear simulations become very different from that in TLM-M, particularly when many noises occur on several spots of the model domain. This appears consistent with the features of spurious perturbations growing over the oceanic area described in the Fig. 3b of Kleist and Morgan (2005a). Note that the above comparison based on the moist basic state is actually not fair since the TLM we use contains no moist physics while the nonlinear model does have moist physics.

To fully address these issues, we believe that more investigations on the impact of the moist physics to the adjoint model needs to be carried out when both the moist TLM and the moist adjoint model are available.

\section{Summary and future prospects}

In addition to various sensitivity products adopted in DOTSTAR so far, a new sensitivity measurement has been proposed here based on the adjoint model. In short, by appropriately defining the response functions to represent the mean steering flow at the verifying time, we can derive its sensitivity to the flow field at the observing time to help formulate the observation strategy. In particular, a simple vector, the ADSSV with respect to the vorticity field, is proposed to clearly demonstrate the sensitivity to the storm's steering flow, and thus the motion. We believe that ADSSV can lend itself to scientific research in many aspects and can be tested in the field project to help improve the typhoon track prediction.

Subsequent work is being carried out to consolidate this study, and will be presented in forthcoming papers.

1) Linearity test and impact of the moist-physics adjoint model:

The results from the linearity test in section 5 starting with the perturbation wind of $1 \mathrm{~m} \mathrm{~s}^{-1}$ shows that the linear assumption remains valid for the 36-h evolution of the MM5 adjoint modeling system with dry physics. It is worth noting that these experiments are only for cases started with a small perturbation at a single grid point. It is consistent with $\mathrm{Mu}$ and Zhang (2006) that the linear assumption works 


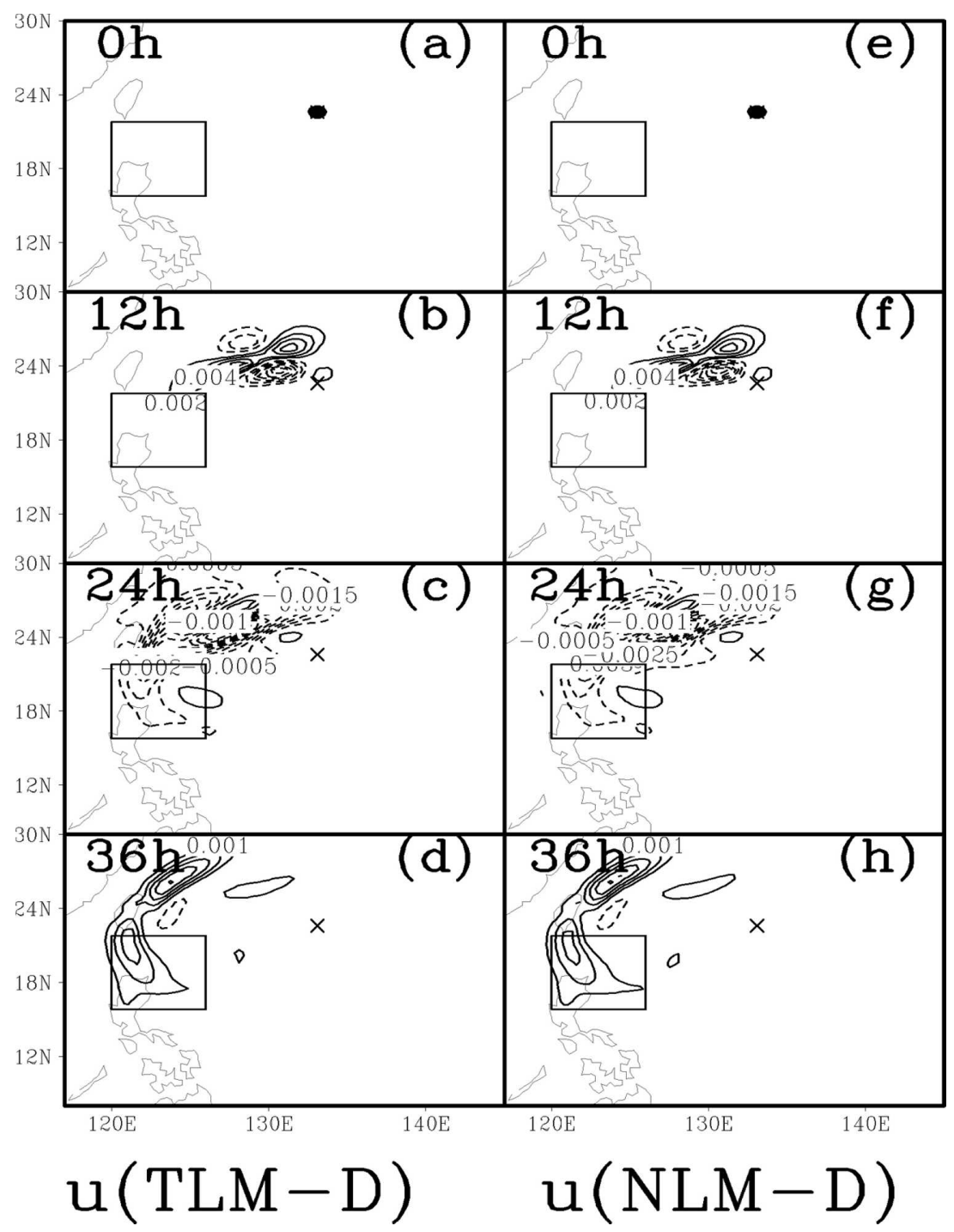

FIG. 13. (a)-(d) Zonal component $(u)$ of the wind perturbation (contour interval of $0.0005 \mathrm{~m} \mathrm{~s}^{-1}$, with the negative value in dashed line) on $\sigma=0.775$ calculated from TLM-D at $0,12,24$, and $36 \mathrm{~h}$, respectively; (e)-(h) same as (a)-(d), but for the difference between two nonlinear dry forecasts (NLM-D). The $\times$ symbol shows the location of the initial perturbation point, and the box represents the verifying area of the adjoint sensitivity.

well for this type of small perturbation. Therefore, more experiments need to be conducted to further examine the impact of perturbations with different magnitudes and patterns (instead of a single grid point) on the validity of the linear assumption.

Note that the adjoint model employed here does not include the moist physics. Although the moist process is definitely critical to the development of the tropical cyclone system, in line with the argument by Peng and Reynolds (2006), we also believe that the tropical cyclone movement is mainly controlled by the large-scale flow field and is less likely related to the moist physics. This hypothesis can be tested using the moist version of the adjoint model, when it is available in the future.

2) Impact study:

The above validation study of the binary interaction between Fengshen and Fungwong indicates that the ADSSV can well represent the signal of the oneway binary interaction process. Besides the binary interaction, the ADSSV can also be used to show how the critical weather system affects the typhoon motion, such as the impact of the approaching trough. To validate the sensitivity derived from the adjoint modeling system in more details, following the Langland et al. (2002) and Kleist and Morgan 


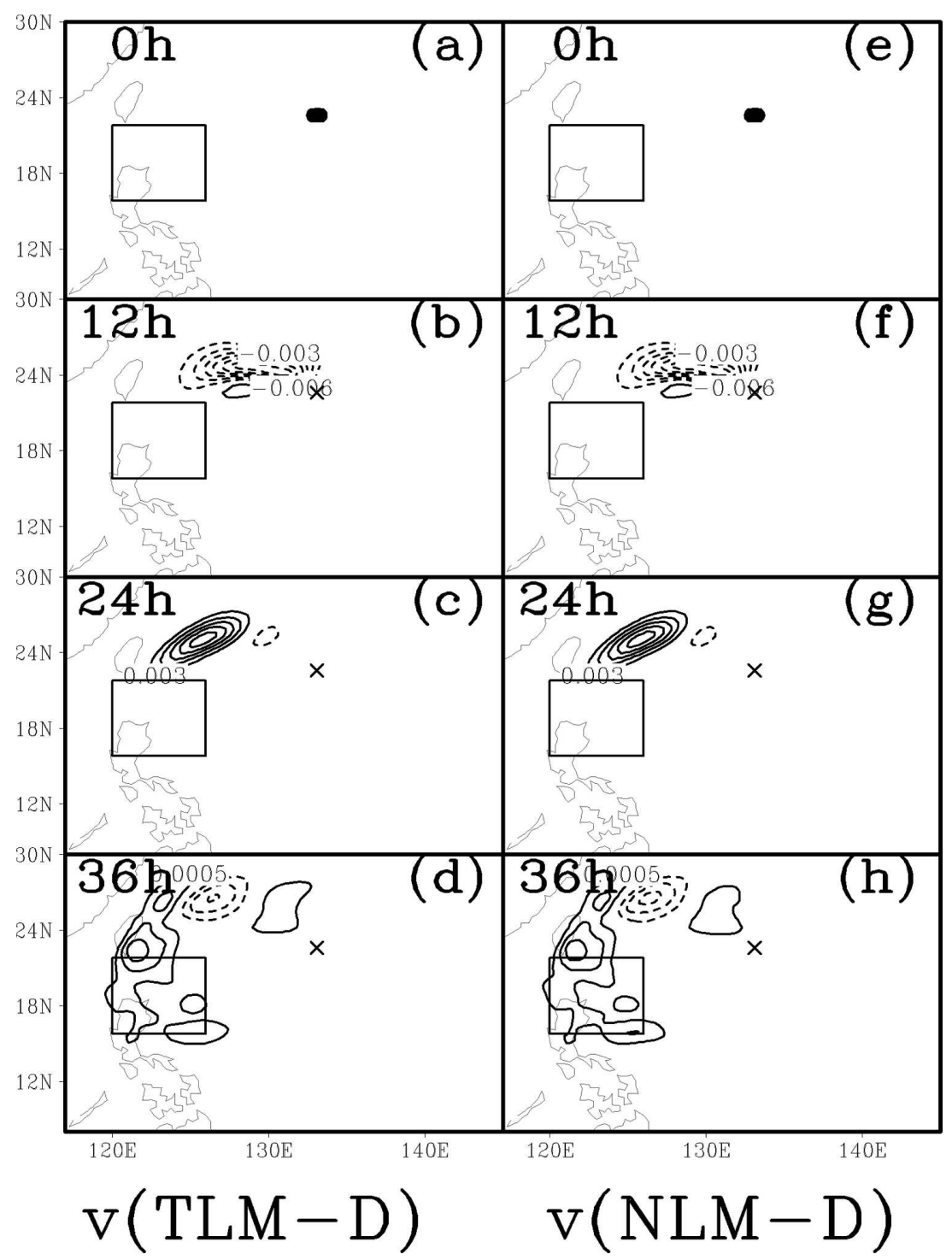

FIG. 14. Same as Fig. 13, but for the meridional wind (v).

(2005b), we also plan to design other experiments, such as to systematically perturb the wind (vorticity) fields in the initial time (such as those in the area with large or small magnitude of ADSSV), and investigate the response to the simulated typhoon track.

3) Application of the ADSSV method to other adjoint modeling systems:

Besides the MM5 adjoint modeling system, there are other adjoint models, such as NOGAPS. How the ADSSV method will appear in different modeling systems is an interesting issue worth further study.

4) Operation in the field program:

While the above task continues, starting from
2005, the updated method (using ADSSV) has been implemented for real-time use in DOTSTAR, as well as for Atlantic hurricanes (Wu 2006; Etherton et al. 2006). A longer period of model integration time is needed because the DOTSTAR operation would require lead time of at least $48 \mathrm{~h}$. The preliminary test is showing consistent results when we run the model for up to $96 \mathrm{~h}$, thus indicating the feasibility of the current system used in DOTSTAR. We believe that using the method of ADSSV in DOTSTAR (Wu et al. 2007) and in the Atlantic (Etherton et al. 2006; including ADSSV comparison with other targeted observation techniques) will shed new light on the targeted observations for tropical cyclones. 


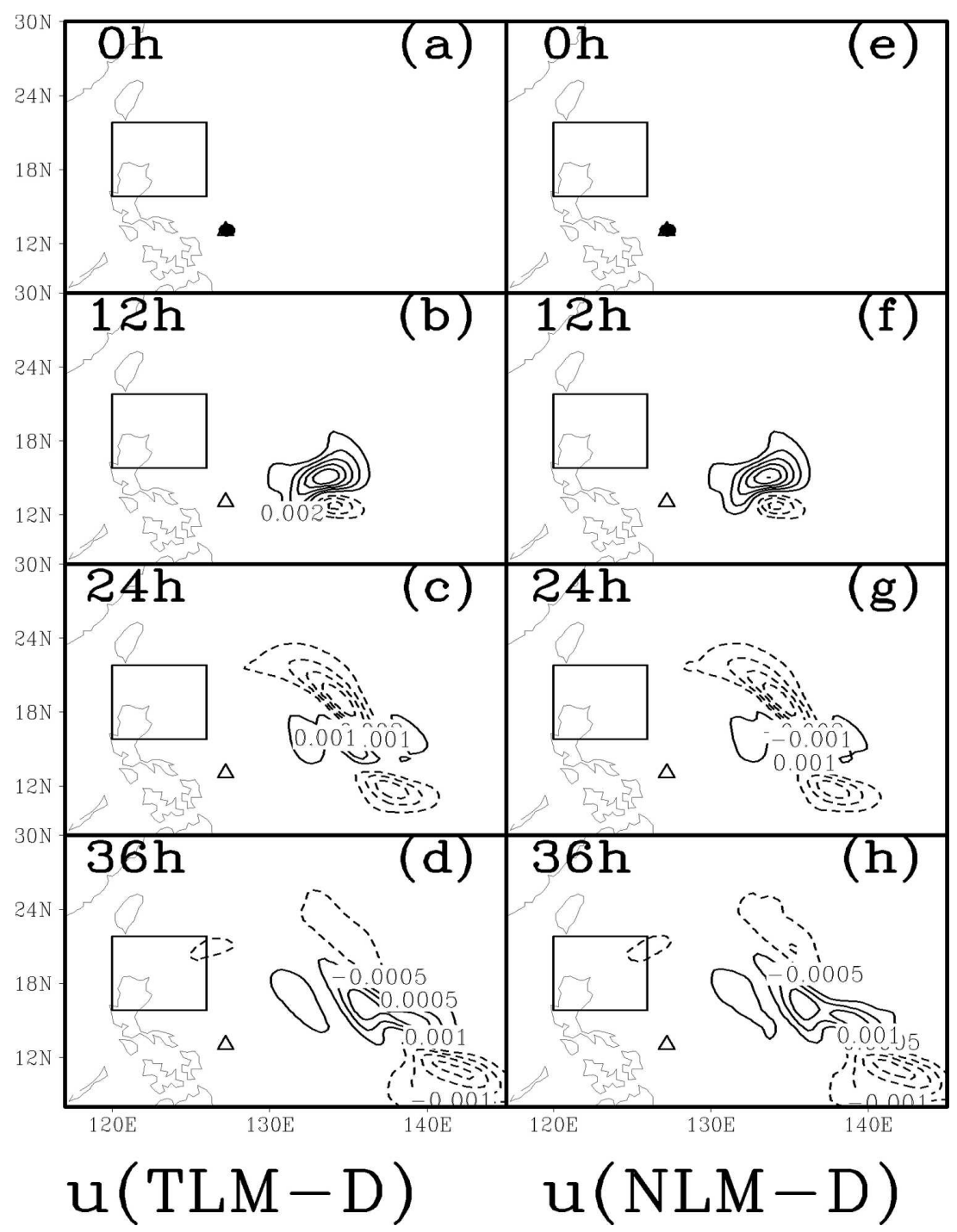

FIG. 15. Same as Fig. 13, but with a different initial perturbation location indicated as the triangle sign.

Acknowledgments. The work is supported through the National Science Council of Taiwan by Grants NSC92-2119-M-002-009-AP1 and NSC93-2119-M-002013-AP1, the Office of Naval Research Grant N0001405-1-0672, and MOTC-CWB-95-6M-03. The authors wish to thank Xiaolei Zou, Mu Mu, Michael Morgan, Seon Park, Hyun Mee Kim, and Chung-I Tseng for their helpful suggestions.

\section{REFERENCES}

Aberson, S. D., 2003: Targeted observations to improve operational tropical cyclone track forecast guidance. Mon. Wea. Rev., 131, 1613-1628.

Chan, J. C.-L., and W. M. Gray, 1982: Tropical cyclone movement and surrounding flow relationship. Mon. Wea. Rev., 110, 1354-1376.

Errico, R. M., 1997: What is an adjoint model? Bull. Amer. Meteor. Soc., 78, 2577-2591.

Etherton, B., C.-C. Wu, S. J. Majumdar, and S. D. Aberson, 2006: A comparison of targeting techniques for 2005 Atlantic tropical cyclones. Preprints, 27th Conf. on Hurricanes and Tropical Meteorology, Monterey, CA, Amer. Meteor. Soc., CDROM, P7.3.

Gelaro, R., T. E. Rosmond, and R. Daley, 2002: Singular vector calculations with an analysis error variance metric. Mon. Wea. Rev., 130, 1166-1186.

Huang, W.-P., C.-C. Wu, P.-H. Lin, and K.-H. Chou, 2006: The impact of the dropwindsonde data from DOTSTAR on the prediction of Typhoon Conson (2004). Preprints, 27th Conf. on Hurricanes and Tropical Meteorology, Monterey, CA, Amer. Meteor. Soc., CD-ROM, 10A.5. 
Kleist, D. T., and M. C. Morgan, 2005a: Interpretation of the structure and evolution of adjoint-derived forecast sensitivity gradients. Mon. Wea. Rev., 133, 466-484.

— , and — 2005b: Application of adjoint-derived forecast sensitivities to the 24-25 January 2000 U.S. East Coast snowstorm. Mon. Wea. Rev., 133, 3148-3175.

Langland, R. H., M. A. Shapiro, and R. Gelaro, 2002: Initial condition sensitivity and error growth in forecasts of the 25 January 2000 east coast snowstorm. Mon. Wea. Rev., 130, 957-974.

Majumdar, S. J., C. H. Bishop, R. Buizza, and R. Gelaro, 2002: A comparison of ensemble-transform Kalman-filter targeting guidance with ECMWF and NRL total-energy singularvector guidance. Quart. J. Roy. Meteor. Soc., 128, 2527-2549.

—, S. D. Aberson, C. H. Bishop, R. Buizza, M. S. Peng, and C. A. Reynolds, 2006: A comparison of adaptive observing guidance for Atlantic tropical cyclones. Mon. Wea. Rev., 134, 2354-2372.

Mu, M., and Z. Zhang, 2006: Conditional nonlinear optimal perturbations of a two-dimensional quasigeostrophic model. $J$. Atmos. Sci., 63, 1587-1604.

Neumann, C. J., 1979: On the use of deep-layer mean geopotential height fields in statistical prediction of tropical cyclone motion. Preprints, Sixth Conf. on Probability and Statistics in Atmosphere Sciences, Banff, AB, Canada, Amer. Meteor. Soc., 32-38.

Palmer, T. N., R. Gelaro, J. Barkmeijer, and R. Buizza, 1998: Singular vectors, metrics, and adaptive observations. J. Atmos. Sci., 55, 633-653.

Peng, M. S., and C. A. Reynolds, 2006: Sensitivity of tropical cyclone forecasts as revealed by singular vectors. J. Atmos. Sci., 63, 2508-2528.

Rohaly, G. D., R. H. Langland, and R. Gelaro, 1998: Identifying regions where the forecast of tropical cyclone tracks is most sensitive to initial condition uncertainty using adjoint meth- ods. Preprints, 12th Conf. on Numerical Weather Prediction, Phoenix, AZ, Amer. Meteor. Soc., 337-340.

Rosmond, T. E., 1997: A technical description of the NRL adjoint model system. Naval Research Laboratory Tech. Doc. NRL MR/7532/97/7230, 62 pp. [Available from Naval Research Laboratory, Monterey, CA 93943-5502.]

Wu, C.-C., 2006: Targeted observation and data assimilation in track prediction. Proc. Sixth Int. Workshop on Tropical Cyclones, San Jose, Costa Rica, WMO/CAS/WWW, 409-423.

— look at the binary interaction: Potential vorticity diagnosis of the unusual southward movement of Typhoon Bopha (2000) and its interaction with Typhoon Saomai (2000). Mon. Wea. Rev., 131, 1289-1300.

— phoon Surveillance near the Taiwan Region (DOTSTAR): An overview. Bull. Amer. Meteor. Soc., 86, 787-790.

- K.-H. Chou, Y. Wang, and Y.-H. Kuo, 2006: Tropical cyclone initialization and prediction based on four-dimensional variational data assimilation. J. Atmos. Sci., 63, 2383-2395.

— - - P.-H. Lin, S. D. Aberson, M. S. Peng, and T. Nakazawa, 2007: The impact of dropwindsonde data on typhoon track forecasts in DOTSTAR. Wea. Forecasting, in press.

Yang, C.-C., and C.-C. Wu, 2004: Binary interaction between Typhoons Fengshen (2002) and Fungwong (2002) based on the potential vorticity diagnosis. Preprints, 26th Conf. on Hurricanes and Tropical Meteorology, Miami, FL, Amer. Meteor. Soc., 663-664.

Zou, X., F. Vandenberghe, M. Pondeca, and Y.-H. Kuo, 1997: Introduction to adjoint techniques and the MM5 adjoint modeling system. NCAR Tech. Note, NCAR/TN-435+STR, 110 pp. [Available from NCAR, P.O. Box 3000, Boulder, CO 80307-3000.] 